There was no significant difference in measured IMT or plaque between patients in LLDAS-50 and those not in LLDAS-50 for the first year after baseline. However, patients in LLDAS-50 were significantly less likely to have major cardiac events (major stroke, myocardial infarction, positive stress test, angioplasty or percutaneous coronary intervention) or death compared with patients who were not in LLDAS-50, $17.1 \%$ and $31.4 \%$, respectively $(p=0.01)$.

Conclusions With regard to damage progression, there was significantly less damage at 3 and 5 years among those in LLDAS $50 \%$ of the time during the first year after cohort entry. Interestingly, although there were no differences between IMT, presence of carotid plaque, or plaque progression at any of the three time points, there was a statistically significant difference in number of cardiovascular events or deaths in the LLDAS-50 group. This supports LLDAS as a valid predictor of lower overall and cardiovascular damage in SLE patients.

Funding Source(s): NCATS Grant No: UL1TR00188

NIH Grant No: 5R01AR063754-02

Correlation Between LLDAS and Cardiovascular Events or Death. Patients in LLDAS 50\% of the time suffer from significantly fewer cardiovascular events or deaths than their nonLLDAS counterparts.

\section{DECREASED EXPRESSION OF RENAL MIR-127-3P CONTRIBUTES TO THE OVERACTIVATION OF INTERFERON SIGNALING PATHWAY IN THE KIDNEY OF LUPUS NEPHRITIS}

${ }^{1}$ Bo Qu*, 'Lingling Wu, 'Xiao Han, 'Le Xiong, 'Zhihua Yin, ${ }^{2} Z$ hizhong Ye, ${ }^{1}$ Nan Shen. 'Department of Rheumatology, Renji Hospital, Shanghai Jiao Tong University School of Medicine; ${ }^{2}$ Shenzhen Futian Hospital for Rheumatic Diseases

\subsection{6/lupus-2019-Ism.177}

Background Lupus nephritis (LN) is one of the most serious manifestations of systemic lupus erythematosus (SLE). Overactivation of type I interferon (IFN) signaling pathway is associated with LN pathogenesis, since overexpression of IFN stimulated genes (ISGs) has been found in the kidney of LN and deficiency of IFN receptor alleviates nephritis in lupus prone mice. Abnormal expression of microRNAs in renal tissues is linked to the pathogenesis of LN. However, the functions of these microRNAs and mechanisms for them to participate in the development of LN are largely unknown. In this study, we aimed to investigate the role of $\mathrm{LN}$-associated renal microRNAs in the overactivation of IFN signaling pathway in the kidney of LN.

Methods microRNA expression was measured by Taqman assay. Interferon-stimulated response element (ISRE)-luciferase reporter assay and western blotting were used to investigate the function of candidate microRNAs in IFN signaling pathway. mRNA expression was measured by SYBR green assay. Gene expression profile was done by microarray. Agomir and antagomir (chemical modified microRNA mimics and inhibitors) of the candidate microRNA was used to perform gain and loss of function experiments. Pristane induced lupus mouse model and NZB/NZW F1 mice were used to investigate the in vivo function of the candidate microRNA.

Results Among revolutionary conserved differentially expressed renal microRNAs in LN, miR-127-3 p, which was reduced in the kidney biopsies of LN patients, inhibited IFN induced ISRE mediated expression of luciferase reporter gene, as well as the phosphorylation of STAT1 and STAT2. By microarray, we revealed that most of the ISGs were inhibited by miR-127-3 $p$ in IFN stimulated Hela cells. Consistently, loss of function of miR-127-3 $\mathrm{p}$ augmented IFN response in human primary renal mesangial cells, with enhanced ISRE mediated expression of reporter gene, phosphorylation of STAT2 and ISGs expression. Further, we identified JAK1, the upstream tyrosine kinase of STAT1 and STAT2, as a novel target of miR-127-3 p. In vivo administration of miR-127-3 $\mathrm{p}$ agomir reduced ISGs expression and alleviated pulmonary hemorrhage induced by pristane in B6 mice and proteinuria in NZB/NZW F1 mice.

Conclusions Our study shows miR-127-3 p can inhibit IFN signaling by targeting JAK1. Decreased expression of miR$127-3 \mathrm{p}$ in the kidney contributes to the overactivated IFN response in LN. Subsequent mouse model studies indicate the therapeutic potential of miR-127-3 $\mathrm{p}$ in treating lupus associated organ damage.

Funding Source(s): This work was supported by the National Basic Research Program of China (973 program) grant 2014 CB541902 and the National Natural Science Foundation of China (No.81401331).

\section{PHASE 2 TRIAL OF INDUCTION THERAPY WITH ANTI- CD20 (RITUXIMAB) FOLLOWED BY MAINTENANCE THERAPY WITH ANTI-BAFF (BELIMUMAB) IN PATIENTS WITH ACTIVE LUPUS NEPHRITIS}

${ }^{1}$ Cynthia Aranow*, ${ }^{2}$ Maria C DallEra, ${ }^{3}$ Margaret Byron, ${ }^{4}$ Linna Ding, ${ }^{5}$ Dawn Smilek, ${ }^{1}$ Betty Diamond, ${ }^{6}$ David Wofsy. ${ }^{1}$ Feinstein Institute for Medical Research; ${ }^{2}$ UC San Francisco; ${ }^{3}$ Rho Federal Systems, Inc; ${ }^{4}$ National Institute of Allergy and Infectious Diseases, DAIT; ${ }^{5}$ Immune Tolerance Network; ${ }^{6}$ Russell/Engleman Rheumatology Research Center, University of California, San Francisco, CA, USA

10.1136/lupus-2019-Ism.178

Background Despite case series suggesting efficacy, controlled trials of anti-CD20 in lupus and lupus nephritis (LN) have not meet their primary endpoints (Arthritis Rheum $2012 ; 64: 1215$ and $2013 ; 65: 2368)$. A potential explanation is the observation that serum BAFF levels are elevated after treatment with rituximab and may lead to disease flare by facilitating maturation of and re-population with autoreactive B cells. The CALIBRATE study (NCT 02260934) was designed to test this hypothesis, to determine whether addition of anti-BAFF (belimumab) could enhance the clinical effects of anti-CD20 (rituximab), and assess safety of the combination. Methods Forty-three patients with active LN despite conventional treatment were enrolled in a prospective randomized open-label trial that compared two therapeutic strategies. All subjects received iv rituximab $(1000 \mathrm{mg})$, CTX $(750 \mathrm{mg})$, and methylprednisolone $(100 \mathrm{mg})$ at wks 0 and 2 , followed by $40 \mathrm{mg} / \mathrm{d}$ prednisone with taper to $10 \mathrm{mg} / \mathrm{d}$ by wk 12 . At wk 4 , subjects were randomized to belimumab $(10 \mathrm{mg} / \mathrm{kg}$ iv at wks $4,6,8$ and then every 4 wks) plus prednisone $(n=21)$ (RCB) or prednisone alone $(\mathrm{RC})(\mathrm{n}=22)$. Complete response $(\mathrm{CR})$ was defined as: (i) urine protein:creatinine ratio (UPCR) $<0.5$; (ii) eGFR 120 or, if $<120$, eGFR $>80 \%$ of screening; and (iii) prednisone dose of $10 \mathrm{mg} / \mathrm{d}$. Partial response (PR) differed only in the UPCR criterion ( $>50 \%$ reduction).

Results The clinical outcome at wk 48 was similar in both groups: CR was $38 \%$ in the belimumab group (RCB) and $32 \%$ in the control group (RC). The frequency of subjects with serious infections was also similar between groups. B cell depletion occurred in both groups by wk 12, but the pace of repopulation was delayed in the RCB group. However, median IgG levels remained within the normal range in both 